\title{
CARTAN STRUCTURES ON CONTACT MANIFOLDS \\ BY
}

G. BURDET AND M. PERRIN ${ }^{1}$

\begin{abstract}
Owing to the existence of a dilatation generator of eigenvalues $\pm 2, \pm 1$, 0 the symplectic Lie algebra is considered as a $|2|$-graded Lie algebra. The corresponding decomposition of the symplectic group $\operatorname{Sp}(2(n+1), \mathbf{R})$ makes the semidirect product (denoted $L^{9}$ ) of the $(2 n+1)$-dimensional Weyl group by the conformal symplectic group $\operatorname{CSp}(2 n, \mathbf{R})$ appear as a privileged subgroup and permits one to construct a $2 n+1$-dimensional homogeneous space possessing a natural contact form. Then $\operatorname{Sp}(2(n+1), \mathbf{R})$-valued Cartan connections on a $L^{0}$. principal fibre bundle over a $2 n+1$-dimensional manifold $B_{2 n+1}$ are constructed and called symplectic Cartan connections. The conditions for obtaining a unique symplectic Cartan connection are given. The existence of this unique Cartan connection is used to define the notion of contact structure over $B_{2 n+1}$ and it is shown that any $L^{0}$-structure of degree 2 over $B_{2 n+1}$ can be considered as a contact structure on it. Moreover it is shown that a contact structure can be associated in a canonical way to any contact manifold.
\end{abstract}

Introduction. In a series of classical papers [2] E. Cartan constructed various spaces with infinitesimal connections, in fact differentiable manifolds whose tangent space attached to each point is a homogeneous space $G / H$, with a possibility of connecting tangent spaces at different neighbouring points. Examples studied were spaces with affine, projective and conformal connections whose tangent spaces are, respectively, isomorphic to $E(n) / S O(n)(E(n)$ being the Euclidean group) $\operatorname{PGL}(n+1, \mathbf{R}) /\left(\mathbf{R}^{n}\right.$ (s) $\left.(\operatorname{SL}(n, \mathbf{R}) \times \mathbf{R})\right)(\operatorname{PGL}(n, \mathbf{R})$ being the projective linear group) and $O(n+1,1) /\left(\mathbf{R}^{n}\right.$ (s) $\left.\left(O(n) \times \mathbf{R}^{+}\right)\right)$. In the language of fibre bundles, connections arising in this way have been called Cartan connections [3] and a precise description of the spaces constructed in [2] has been given in [4] by using frames of the second order to introduce the notion of Cartan structure.

Then a Cartan structure $P$ appears as an $\boldsymbol{H}$-structure of degree 2 over a manifold $B$ (with $\operatorname{dim} B=\operatorname{dim} G / H$ ) equipped with a uniquely defined torsion-free Cartan connection.

There are many works concerning the situation where $G$ is semisimple, its Lie algebra $g$ is a semisimple $|1|$-graded Lie algebra, i.e. it can be written as $g=g_{-1}+$ $g_{0}+g_{1}$, and $g_{-1}+g_{0}$ is the Lie algebra of $H$ (see the references quoted in [7]).

Among the established results we want to point out the following:

(1) $H$ is a semidirect product $G_{1}$ (5) $G_{0}$ where $G_{0}$ is the normalizer of $\mathrm{g}_{0}$ in $H$ and $G_{1}$ is Abelian with Lie algebra $g_{-1}$.

(2) $G_{0}$ is the linear isotropy group of the homogeneous space $G / H$.

(3) $G \rightarrow G / H$ can be naturally considered as an $H$-structure of degree 2 .

Received by the editors April 14, 1980 and, in revised form, July 29, 1980.

1980 Mathematics Subject Classification. Primary 53C05, 53C10, 53B15; Secondary 17B70.

${ }^{1}$ Aide Individuelle du C.N.R.S. no. 032795. 
(4) Any given torsion-free $G_{0}$-connection on a $G_{0}$-structure (of degree 1 ) determines an associated $\boldsymbol{H}$-structure of degree 2 and there is a notion of $\boldsymbol{H}$-equivalence between such $G_{0}$-connections.

(5) The Lie group $\operatorname{Aut}(P, \omega)$ of automorphisms of the geometric structure $(P, \omega)$ attains its maximum dimension if and only if the 2-form (curvature and torsion) of $\omega$ vanishes identically.

Finally all the $|1|$-graded semisimple Lie algebras have been classified.

Note that both classical geometries, the projective and the conformal ones, fall into this scheme, and that the conformal equivalence classes of Riemannian metrics on $B$ are in a natural one-to-one correspondence with conformal structures.

The purpose of this paper is to present an example of a Cartan structure which does not belong to the above described scheme since it deals with a $|2|$-graded Lie algebra $g=g_{-2}+g_{-1}+g_{0}+g_{1}+g_{2}$. $G$ is the symplectic group $\operatorname{Sp}(2(n+1), \mathbf{R})$ and $H$ now appears as a one-dimensional central extension $L^{0}$ of the linear isotropy group.

Again it is possible to consider $L^{0}$-structures of degree 2 with symplectic valued Cartan connections. Then it is shown that the Lie group of automorphisms of this geometric structure can attain its maximum dimension with a nonzero particular component of the two-form of the connection.

The basis manifold is odd dimensional for $\operatorname{dim} G / H=2 n+1$, and an $L^{0}$-structure of degree 2 endowed with a uniquely defined symplectic torsionless Cartan connection is called a contact structure because it can be shown that, over a contact manifold, an $L^{0}$-structure of degree 2 can be associated in a canonical way.

For convenience let us recall the definition of a contact manifold [5]. Let $C$ be a differentiable manifold of odd dimension $2 n+1$. If there is defined on $C$ an open covering $\left\{\mathscr{U}_{j}\right\}$ together with a system of one-forms $\alpha=\left\{\alpha_{j}\right\}$ such that $\alpha_{j} \wedge\left(d \alpha_{j}\right)^{n}$ $\neq 0$ everywhere on $\mathscr{\vartheta}_{j}$, and possessing a suitable adjusting property on $\mathcal{U}_{j} \cap \mathcal{U}_{i}$, then $(C, \alpha)$ is said to be a contact manifold with $\alpha$ as contact form.

$\left(\mathcal{Q}_{j}, \alpha_{j}\right)$ and $\left(\mathscr{U}_{i}, \alpha_{i}\right)$ are said to be equivalent if $\alpha_{i}=\varphi_{i j} \alpha_{j}$ on $\mathscr{U}_{j} \cap \mathscr{U}_{i}$, where $\varphi_{i j}$ is some nonvanishing function on $\mathscr{U}_{j} \cap \mathcal{Q}_{i}$. A transformation $f$ of $C$ is called a contact transformation if $\left(f^{-1} \mathcal{Q}_{j}, f^{*} \alpha_{j}\right)$ and $\left(\mathcal{Q}_{j}, \alpha_{j}\right)$ are equivalent.

Following the approach of [4] and references therein, this paper is organized as follows:

$\S 1$ deals with a symmetric decomposition of the symplectic group which leads to the wanted graded decomposition of the symplectic Lie algebra and to a homogeneous space possessing a natural contact form.

Cartan connections are defined in $\$ 2$. Symplectic Cartan connections are studied as the properties of their automorphism group. Additional properties to obtain a unique symplectic Cartan connection are also exhibited.

In $\$ 3$ we recall the notion of Cartan structure, and contact Cartan structures over odd-dimensional manifolds are defined. Then contact structures associated with contact manifolds are studied.

Note that the particular Cartan connections here studied are called symplectic Cartan connections (their one-forms are $\operatorname{sp}(2(n+1), \mathbf{R})$-valued) and not contact 
connections. This denomination is justified by the fact that it is also possible to construct over a contact manifold another type of second order structure endowed with an $\operatorname{so}(p+2, q+2)$-valued connection and whose linear isotropy group is a subgroup of the previous one. This is another example of a geometrical structure corresponding to a $|2|$-graded semisimple Lie algebra.

This paper is an enlarged version of a previous one and we would like to thank the referee for his valuable comments.

1. A homogeneous space with a natural contact form related to a symmetric decomposition of the symplectic group.

1.1 Let us consider the symplectic group $\operatorname{Sp}(2(n+1), \mathbf{R})$ realized as the set $L$ of matrices $m \in \operatorname{Gl}(2(n+1), \mathbf{R})$ satisfying $m J^{\prime} ' m=J^{\prime}$, where $J^{\prime}$ is a skew symmetric matrix such that $J^{\prime 2}=-\mathbf{1}_{2(n+1)}$.

We need a particular decomposition of $L$ obtained by setting

$$
J^{\prime}=\left(\begin{array}{ccc}
0 & 0 & -1 \\
0 & J & 0 \\
1 & 0 & 0
\end{array}\right),
$$

where $J$ is also a skew symmetric matrix such that $J^{2}=-1_{2 n}$, and by introducing the set $L_{e}=\left\{h k h^{*}\right\}$ where

$$
\begin{aligned}
& h \in W_{n}=\left\{h \in M_{2(n+1)}(\mathbf{R}) \mid h\left(\bar{v}, v_{0}\right)=\left(\begin{array}{ccc}
1 & -{ }^{t} \bar{v} J & v_{0} \\
0 & \mathbf{1}_{2 n} & \bar{v} \\
0 & 0 & 1
\end{array}\right),\right. \\
& \left.v_{0} \in \mathbf{R}, \bar{v} \text { : column } 2 n \times 1 \text { matrix }\right\} \text {, } \\
& h^{*} \in W_{n}^{*}=\left\{h^{*} \in M_{2(n+1)}(\mathbf{R}) \mid h^{*}\left(\underline{u}, u_{0}\right)=\left(\begin{array}{ccc}
1 & 0 & 0 \\
J^{t} \underline{u} & 1_{2 n} & 0 \\
u_{0} & \underline{u} & 1
\end{array}\right),\right. \\
& \left.u_{0} \in \mathbf{R}, \underline{u}: \text { row } 1 \times 2 n \text { matrix }\right\} \text {, } \\
& k \in K=\left\{\left(\begin{array}{ccc}
r & 0 & 0 \\
0 & l & 0 \\
0 & 0 & r^{-1}
\end{array}\right) \mid r \in \dot{\mathbf{R}}, l \in \mathrm{Sp}(2 n, \mathbf{R}) \text {, i.e. } J^{\prime} l=J\right\} .
\end{aligned}
$$

$L_{e}$ is an open neighbourhood of the identity and is dense into $L$. We shall take $\left(\bar{v}, v_{0}, l, r, \underline{u}, u_{0}\right)$ as a coordinate system in $L_{e}$. Let us note that:

(i) $K$ is a subgroup isomorphic to $\operatorname{Sp}(2 n, \mathbf{R})$ (3) $\dot{\mathbf{R}}=\operatorname{CSp}(2 n, \mathbf{R})$, the so-called conformal symplectic group;

(ii) both $W_{n}$ and $W_{n}^{*}$ are coverings $\mathbf{R}^{2 n} \times \mathbf{R}$ of the Weyl-Heisenberg group $\mathbf{R}^{2 n} \times S^{1}$; they are conjugated for instance via the linear involutive inner automorphism $J^{\prime} h\left(\bar{v}, v_{0}\right) J^{\prime-1}=h^{*}\left(-{ }^{t} \bar{v},-v_{0}\right)$. Their group law is given by 


$$
\left(\bar{v}, v_{0}\right)\left(\bar{v}^{\prime}, v_{0}^{\prime}\right)=\left(\bar{v}+\bar{v}^{\prime}, v_{0}+v_{0}^{\prime}-{ }^{t} \bar{v} J \bar{v}^{\prime}\right)
$$

(iii) the sets $\{h k\}$ and $\left\{k h^{*}\right\}$ define two semidirect products isomorphic to the semidirect product of $\operatorname{CSp}(2 n, \mathbf{R})$ acting on the covering of the Weyl-Heisenberg group.

Then let $L^{0}$ be the set $\left\{k h^{*}\right\}$ parametrized by $\left(\overline{0}, 0, l, r, \underline{u}, u_{0}\right) . L^{0}$ is dense into the semidirect product $W_{n}^{*}$ (S) $\operatorname{CSp}(2 n, \mathbf{R})$ and its multiplication law is given by

$$
\begin{aligned}
\left(\overline{0}, 0, l, r, \underline{u}, u_{0}\right) & \left(\overline{0}, 0, l^{\prime}, r^{\prime}, \underline{u}^{\prime}, u_{0}^{\prime}\right) \\
= & \left(\overline{0}, 0, l l^{\prime}, r r^{\prime}, \underline{u}^{\prime}+r^{\prime} \underline{u} l^{\prime}, u_{0}^{\prime}+r^{\prime 2} u_{0}+\frac{1}{2} r^{\prime} \underline{u} l^{\prime} J^{\prime} \underline{u}^{\prime}\right) .
\end{aligned}
$$

1.2 The Maurer-Cartan form on the symplectic group and the symplectic Lie algebra. For any $m \in L_{e}$ such that $m^{-1}$ exists, let us consider the differential 1-form $\omega=m^{-1} d m$ which satisfies the structure equation of Maurer-Cartan, $d \omega=-\omega \wedge \omega$. By differentiating the relation $m J^{\prime} t m=J^{\prime}$ one gets $\omega J^{\prime}+J^{\prime}{ }^{t} \omega=$ 0 , so that $\omega$ can be written as

$$
\omega=\left(\begin{array}{ccc}
-w_{0^{\prime}}^{\sigma^{\prime}} & -{ }^{t} \bar{w}_{0} J & w_{0}^{0} \\
J^{t} \underline{w}^{\sigma} & w & \bar{w}_{\sigma} \\
w_{0}^{\sigma^{\prime}} & \underline{w}^{\sigma} & w_{0}^{\sigma}
\end{array}\right)
$$

where

(i) $\bar{w}_{0}$ is written as a column $2 n \times 1$ matrix $\bar{w}_{0}=\left\{w_{0}^{j}\right\}, j \in[1,2 n]$, its transpose being the row ${ }^{\prime} \bar{w}_{0}$;

(ii) $\underline{w}^{0}$ is written as a row $1 \times 2 n$ matrix $\underline{w}^{0}=\left\{w_{j}^{0}\right\}, j \in[1,2 n]$, its transpose being the column ${ }^{t} \underline{w}^{0}$;

(iii) $w$ is a $2 n \times 2 n$ matrix which can be written in block form as

$$
\left(\begin{array}{cc}
R & S \\
T & -{ }^{t} R
\end{array}\right) \text { if } J=\left(\begin{array}{cc}
0 & 1_{n} \\
-1_{n} & 0
\end{array}\right) .
$$

$S$ and $T$ are symmetric matrices:

$$
S=\left\{w_{j+n}^{k}\right\}={ }^{t} S \text { and } T=\left\{w_{j}^{k+n}\right\}={ }^{t} T
$$

$R$ is any $n \times n$ matrix $\left\{w_{j}^{k}\right\}, j, k \in[1, n]$.

By setting $\tilde{w}_{j}^{k}=w_{j}^{k}-\delta_{j}^{k} w_{0^{\prime}}^{0}$, and with the agreements $w_{i+n}^{0}=-w_{i-n}^{0}$ and $w_{0}^{i+n}=$ $-w_{0^{\prime}}^{i-n}$ for $i \in[n+1,2 n]$, the Maurer-Cartan structure equations are explicitly written as follows:

$$
\begin{aligned}
& d w_{0^{\prime}}^{j}=w_{0^{\prime}}^{0} \wedge w_{n+j}^{\alpha}-\sum \tilde{w}_{l}^{j} \wedge w_{0}^{l} ; \\
& d w_{0^{\prime}}^{0}=-2 w_{0^{\prime}}^{0} \wedge w_{0^{\prime}}^{\sigma}-\sum w_{0}^{l+n} \wedge w_{0}^{l} ; \\
& d \tilde{w}_{j}^{k}=w_{j}^{\sigma} \wedge w_{0^{\prime}}^{k}+w_{0}^{j+n} \wedge w_{k+n}^{\sigma} \\
& -\sum \tilde{w}_{l}^{k} \wedge \tilde{w}_{j}^{l}-\delta_{j}^{k}\left(w_{0}^{0} \wedge w_{0}^{\alpha}-\sum w_{l}^{\alpha} \wedge w_{\alpha}^{l}\right) ; \\
& d w_{0^{\prime}}^{0}=w_{0^{\prime}}^{0} \wedge w_{0}^{0}-\sum w_{l}^{\sigma} \wedge w_{\alpha}^{l} ; \\
& d w_{j}^{\sigma}=w_{0^{\prime}}^{n+j} \wedge w_{0}^{\sigma}-\sum w_{l}^{\sigma} \wedge \tilde{w}_{j}^{l} ;
\end{aligned}
$$




$$
d w_{0}^{\alpha}=-2 w_{0}^{\alpha} \wedge w_{0}^{\sigma}-\sum w_{l}^{\alpha} \wedge w_{l+n}^{\alpha}
$$

where all the sums have to be performed over $l \in[1,2 n]$.

Now let $\left\{E_{\mu}^{\nu} ; \mu, \nu \in\left[0,1, \ldots, 2 n, 0^{\prime}\right]\right\}$ be a basis of the Lie algebra $\operatorname{sp}(2(n+1), \mathbf{R})$ which satisfies

$$
w_{\mu}^{\nu}\left(E_{\rho}^{\lambda}\right)=\delta_{\rho}^{\nu} \delta_{\mu}^{\lambda} .
$$

From (5) one verifies that this basis shows the existence of a symmetric decomposition of $\operatorname{sp}(2(n+1), \mathbf{R})$ which, as a vector space, is given by [1]

$$
\operatorname{sp}(2(n+1), \mathbf{R})=h_{n}+\operatorname{csp}(2 n, \mathbf{R})+h_{n}^{*}:
$$

(i) $h_{n}$ generated by the set $\left\{E_{\mu}^{\sigma}, \mu \in[0,1, \ldots, 2 n]\right\}$ (respectively, $h_{n}^{*}$ generated by $\left.\left\{E_{0}^{\mu}, \mu \in[0,1, \ldots, 2 n]\right\}\right)$ is the $(2 n+1)$-dimensional Heisenberg algebra, the Lie algebra of $W_{n}\left(W_{n}^{*}\right.$ resp.),

(ii) $\operatorname{csp}(2 n, \mathbf{R})=\left\{\tilde{Z} \in \operatorname{gl}(2 n, \mathbf{R}) \mid \tilde{Z} J+J^{t} \tilde{Z}=-2 \lambda J, \lambda \in \dot{\mathbf{R}}\right\}$ is the conformal symplectic algebra which can be written as the direct $\operatorname{sum} \operatorname{sp}(2 n, R) \oplus \dot{\mathbf{R}}$; the symplectic algebra $\operatorname{sp}(2 n, \mathbf{R})(\lambda=0)$ is generated by $\left\{E_{j}^{k}, j, k \in[1,2 n]\right\}$ and $\dot{\mathbf{R}}$ by $E_{0^{\prime}}^{\sigma^{\prime}}$.

Under the action of the dilation generated by $E_{0^{\prime}}^{\sigma}$ the Lie algebra $\operatorname{sp}(2(n+1), \mathbf{R})$ splits into eigensubspaces $\mathrm{g}^{(r)}$ such that

$$
\left[E_{\sigma}^{\alpha}, \mathfrak{g}^{(r)}\right]=r \mathrm{~g}^{(r)}, \quad r=0, \pm 1, \pm 2 ;
$$

hence the decomposition (7) can be refined and the Lie algebra $\operatorname{sp}(2(n+1), \mathbf{R})$ becomes a $|2|$-graded semisimple Lie algebra

$$
\operatorname{sp}(2(n+1), \mathbf{R})=\sum_{r=-2}^{+2} \mathrm{~g}^{(r)}
$$

with $\left[\mathrm{g}^{(r)}, \mathrm{g}^{\left(r^{\prime}\right)}\right] \subseteq \mathrm{g}^{\left(r+r^{\prime}\right)}, \mathrm{g}^{(r)}=0$ if $r>2$ or $r<-2$. Then $h_{n}=\mathrm{g}^{(2)}+\mathrm{g}^{(1)}$, $\operatorname{csp}(2 n, \mathbf{R})=\mathfrak{g}^{(0)}, h_{n}^{*}=\mathrm{g}^{(-1)}+\mathfrak{g}^{(-2)}$.

According to the decomposition (7), any element $z$ of $\operatorname{sp}(2(n+1), \mathbf{R})$ can split as follows:

(i) as a vector space $h_{n}=\mathbf{R}^{2 n}+\mathbf{R}$ and the restriction $\left.z\right|_{h_{n}}$ of $z$ to $h_{n}$ is identified with the couple $\left(\bar{Z}, Z_{0}\right)$ where $\bar{Z}$ is a column $2 n \times 1$ matrix and $Z_{0}$ is proportional to $E_{0}^{\sigma}$; then $\left.z\right|_{n_{n}^{*}}$ consists of the couple $\left(\underline{Z}, Z^{0}\right)$ where $\underline{Z}$ is a row $1 \times 2 n$ matrix and $Z^{0}$ is proportional to $E_{0}^{0}$;

(ii) $\left.z\right|_{\mathrm{sp}(2 n, \mathbf{R})}$ is written as the $2 n \times 2 n$ symplectic matrix $Z$ and we set $Z_{0}=\lambda E_{0}^{\alpha}$; then $\left.z\right|_{\operatorname{csp}(2 n, \mathbf{R})}$ is written as the matrix $\tilde{Z}=Z-\lambda \mathbf{1}_{2 n}$.

Under these identifications the set of structure equations (5) is equivalent to the following set of commutation relations:

$$
\begin{aligned}
{\left[\left(\bar{Z}, Z_{0}\right),\left(\bar{Z}^{\prime}, Z_{0}^{\prime}\right)\right]=} & \left(\overline{0},-2^{t} \bar{Z} J \bar{Z}^{\prime}\right) ; \\
{\left[\left(\underline{Z}, Z^{0}\right),\left(\underline{Z}^{\prime}, Z^{0 \prime}\right)\right]=} & \left(\underline{0}, 2 \underline{Z} J^{\prime} \underline{Z}^{\prime}\right) ; \\
{\left[\left(\bar{Z}, Z_{0}\right),\left(\underline{Z}, Z^{0}\right)\right]=} & \left(-Z_{0} J^{t} \underline{Z}, 0\right)+\left(Z^{0}{ }^{t} J, 0\right)+\bar{Z} \otimes \underline{Z} \\
& +J^{t} \underline{Z} \otimes{ }^{\prime} \bar{Z} J+\left(Z_{0} Z^{0}+(\underline{Z} \bar{Z})\right) \mathbf{1}_{2 n} ;
\end{aligned}
$$




$$
\begin{aligned}
{\left[\tilde{Z}, \tilde{Z}^{\prime}\right] } & =\tilde{Z} \tilde{Z^{\prime}}-\tilde{Z}^{\prime} \tilde{Z}=\left[Z, Z^{\prime}\right] ; \\
{\left[\tilde{Z},\left(\bar{Z}, Z_{0}\right)\right] } & =\left(\tilde{Z} \bar{Z},-2 Z_{0} Z_{0}\right) ; \\
{\left[\left(\underline{Z}, Z^{0}\right), \tilde{Z}\right] } & =\left(\underline{Z} \tilde{Z},-2 Z^{0} Z_{0}\right) ;
\end{aligned}
$$

(10f) corresponds to the semidirect $\operatorname{sum} h_{n}^{*} \quad \square \operatorname{csp}(2 n, \mathbf{R})$ denoted by $\mathfrak{l}^{0}$ which is the Lie algebra of $L^{0}$.

From (7) it is clear that $\mathfrak{l}^{0}$ has a vector space complement in $\mathfrak{l}$ which is the Lie algebra $h_{n}$ and (10c) shows that $h_{n}$ is not invariant under the adjoint action of $\mathfrak{l}^{0}$ so that the decomposition $\mathfrak{l}=\mathfrak{l}^{0}+h_{n}$ is not reductive.

1.3 The natural contact structure on $L_{e} / L^{0}$. Let us consider the connected homogeneous space $L_{e} / L^{0}$. According to the definitions of $L_{e}$ and $L^{0}$ any element $z$ of the left coset $L_{e} / L^{0}$ can be represented by the matrix $h\left(\bar{z}, z_{0}\right)$ defined in (1a) and then identified with $\mathbf{R}^{2 n+1}$. The class of the identity $e \in L_{e}$ in $L_{e} / L^{0}$, i.e. the origin 0 of $L_{e} / L^{0}$, is represented by $1_{2 n+2}$. Then for any $m \in L_{e}$ the left action of $L_{e}$ on $L_{e} / L^{0}$ gives rise to a linear fractional transformation $\left(\bar{z}, z_{0}\right) \mapsto f_{m}\left(\bar{z}, z_{0}\right)=$ $\left(\frac{\hat{z}}{,} \hat{z}_{0}\right)$ given by

$$
\begin{aligned}
\overline{\hat{z}} & =D\left\{\left(l+r^{-1} \bar{v} \otimes \underline{u}\right) \bar{z}+\left(J^{t} \underline{u}+r^{-1} u_{0} \bar{v}\right) z_{0}+r^{-1} \bar{v}\right\} \\
\hat{z}_{0} & =D\left\{\left(r^{-1} v_{0} \underline{u}-{ }^{t} \bar{v} J l\right) \bar{z}+\left(r-\underline{u} l^{-1} \bar{v}+r^{-1} u_{0} v_{0}\right) z_{0}+r^{-1} v_{0}\right\}
\end{aligned}
$$

with $D=r\left(1+\underline{u} \bar{z}+u_{0} z_{0}\right)^{-1}$; then $L^{0}$ is the stabilizer of 0 .

Let us note here that there is a natural representation $\rho$ usually called the linear isotropy representation of $L^{0}$ on the tangent space of $L_{e} / L^{0}$ at the origin 0 . $T_{0}\left(L_{e} / L^{9}\right)=\mathfrak{l} / \mathfrak{l}^{0}=h_{n}$ from the decomposition of $\mathfrak{l}$; then the linear isotropy representation is defined by

$$
\rho(g) X=\operatorname{Ad}(g) X \quad\left(\bmod \mathfrak{l}^{0}\right) \quad \text { for } g \in L^{0} \text { and } X \in h_{n}
$$

and represents $L^{0}$ as a subgroup of $\mathrm{GL}(2 n+1, \mathbf{R})$. This representation is not faithful and possesses a one-dimensional kernel isomorphic to $\mathbf{R}$, the Lie algebra of which is $\mathrm{g}^{(-2)}$. This is due to the fact that $W_{n}(\mathcal{C S p}(2 n, \mathbf{R})$ is a one-dimensional central extension of the inhomogeneous symplectic group $\operatorname{ICSp}(2 n, \mathbf{R})=$ $\mathbf{R}^{2 n}$ (3) $\operatorname{CSp}(2 n, \mathbf{R})$, i.e. the semidirect product of $\mathbf{R}^{2 n}$ by $\operatorname{CSp}(2 n, \mathbf{R})$, corresponding to a chosen section $k$ : one has the following exact sequence:

$$
1 \rightarrow \mathbf{R} \stackrel{i}{\rightarrow} W_{n} \otimes \operatorname{CSp}(2 n, \mathbf{R}) \underset{p}{\stackrel{k}{\leftrightarrows}} \operatorname{ICSp}(2 n, \mathbf{R}) \rightarrow 1
$$

If $\beta$ is the 2 -cocycle of the $\mathbf{R}$-valued $\operatorname{ICSp}(2 n, \mathbf{R})$ cohomology which defines the central extension, then $k$ is an injective homomorphism, such that $p \circ k=$ $\operatorname{Id}_{\operatorname{ICSp}(2 n, \mathbf{R})}$, related to $\beta$ as follows:

$$
k(a) k(b) k(a b)^{-1}=i(\beta(a, b)), \quad a, b \in \operatorname{ICSp}(2 n, \mathbf{R}) .
$$

So $\rho$ is faithful on the image of $k$ only, and $\operatorname{ICSp}(2 n, \mathbf{R})$ is called the linear isotropy group of the homogeneous space $L_{e} / L^{0}$.

Now we want to show that there exists a natural contact structure on $L_{e} / L^{0}$ (see the introduction for the definition of a contact manifold). According to the decomposition of the symplectic algebra $\mathfrak{l}=\mathfrak{l}^{0}+h_{n}$, let us consider the corresponding decomposition of the Maurer-Cartan form written as $\omega=\omega_{\mathfrak{l}^{0}}+\omega_{h_{h}}$, 
where $\omega_{\mathrm{I}^{0}}=\left\{w_{0}^{0}, \underline{w}^{\sigma}, w, w_{0^{\gamma}}^{0}\right\}$ and $\omega_{h_{n}}=\left\{w_{0^{0}}^{0}, \bar{w}_{0^{0}}\right\}$. From (6) one verifies that $\omega_{\mathfrak{l}^{0}}$ is the $\mathfrak{I}^{0}$-component of $\omega$ and $\omega_{h_{n}}$ its $h_{n}$-component. Both of these forms are defined on $L$ but let us notice that the restriction of $\omega_{1^{0}}$ to $L^{0}$ is the Maurer-Cartan form on $L^{0}$, and that the restriction of $\omega_{h_{n}}$ to $W_{n}$ is the Maurer-Cartan form on $W_{n}$. Now we look for the forms which can be defined on $L_{e} / L^{0}$. It is well known that

$$
T_{[g]}^{*}\left(L_{e} / L^{0}\right) \approx\left\{\omega \in T_{g}^{*}\left(L_{e}\right) \mid \omega\left(T_{g}\left(L^{0} g\right)\right)=0\right\} \quad \forall g \in L_{e},
$$

where $L^{0} g$ denotes the left action of $L^{0}$ on $L_{e}$.

It is easy to see that the forms which define a horizontal subspace on $L_{e}$ satisfy this condition and therefore provide a basis for $T_{g}^{*}\left(L_{e} / L^{9}\right)$. Among them, by using (5b), one can verify that the restriction $\alpha^{0}$ to $L_{e} / L^{0}$ of $w_{0}^{0}$ is a contact form, i.e. it satisfies $\alpha^{0} \wedge\left(d \alpha^{0}\right)^{n} \neq 0$ everywhere on $L_{e} / L^{0}$. So the space $L_{e} / L^{0}$ is endowed with a contact structure. With the above given identification $\alpha^{0}$ is given by

$$
\alpha^{0}=d z_{0}+\frac{1}{2}{ }^{t} \bar{z} J d \bar{z}
$$

\section{Symplectic Cartan connections.}

2.1 Cartan connection notion. Let $P(B, H, \pi)$ be a principal fibre bundle over a manifold $B$ with structure group $H$ and projection $\pi$. Let us suppose that $H$ is a connected subgroup of a Lie group $G$ and that $\operatorname{dim} B=\operatorname{dim} G / H$. The group $H$ acts on the left on $G$ by $(h, g) \mapsto h g, h \in H, g \in G$. Hence one can introduce the extension $P^{G}$ of $P$, i.e. the associated fibre bundle $P^{G}=P \times_{H} G$. Moreover let $G$ act on the right on $P^{G}$ as follows: $R_{g^{\prime}}(u \cdot g)=u \cdot\left(g g^{\prime}\right)$ for $u \in P, g$ and $g^{\prime} \in G$; therefore $P^{G}$ is a principal fibre bundle over $B$ with structure group $G$ and the mapping $u \mapsto(u \cdot e), e$ being the identity of $G$, defines an embedding of $P$ into $P^{G}$.

Definition 1. A connection $\Gamma$ in $P^{G}$ is said to be a Cartan connection for $P$ with respect to $G$ if $H_{u_{b}} \cap T_{u_{b}}(P)=\{0\}$, where $u_{b}$ belongs to $P_{b}$, the fibre of $P$ over $b \in B, T_{u_{b}}(P)$ is the tangent space to $P$ at $u_{b}$, and $H_{u_{b}}$ is the horizontal subspace of $T_{u_{b}}\left(P^{G}\right)$ with respect to $\Gamma$.

Let $\omega$ now denote the restriction to $P$ of the connection form of $\Gamma$; then $\omega$ is a differential 1-form on $P$ with value in the Lie algebra $g$ of $G$, the so-called Cartan connection form of $P$. At any point $u$ of $P, \omega_{u}$ defines a linear isomorphism of $T_{u}(P)$ into $g$.

Conversely let $\omega$ be a differential 1 -form on $P$ with value into $g$ satisfying the following properties:

P1. $\omega\left(X^{*}\right)=X$ for every $X$ belonging to $\mathfrak{h}$ the Lie algebra of $H\left(X^{*}\right.$ denoting the fundamental vector field corresponding to $X[6])$,

P2. $R_{h}^{*}(\omega)\left(R_{h}(X)\right)=\operatorname{ad}\left(h^{-1}\right) \omega(X), R_{h}^{*}$ denoting the action on 1-forms of $h \in H$ and $R_{h}$ the right action on $P$, while ad denotes the adjoint representation of $H$ on $g$,

P3. $\omega(X) \neq 0$ for every nonzero vector $X$ on $P$;

Then $\omega$ can be uniquely extended to a usual connection form on $P^{G}$ and the set of properties P1-P3 can be taken as the definition of a Cartan connection on $P$.

The main interest for introducing the notion of Cartan connection lies in the fact that if there exists a Cartan connection for $P$, then $P$ is parallelizable; in other words $P$ admits a 1 -structure or its tangent bundle $T(P)$ is trivializable [7]. 
It is also interesting to recall that the same property can be obtained by introducing the soldering notion as follows [3]. As a subgroup of $G, H$ acts on the right on $P^{G}$, and the orbit manifold $\tilde{P}=H \backslash P^{G}$ can be canonically identified with the associated fibre bundle $\tilde{P}=P \times{ }_{H} G / H$ corresponding to the action on the left of $H$ on $G / H$. Let $\tilde{B}$ be the canonical section $\tilde{B}=\sigma(B)$ where $\sigma: \pi(u) \mapsto u \cdot e^{\prime}$ ( $e^{\prime}$ being the canonical image in $G / H$ of the identity $e$ of $G$ ). Let us consider $V(\tilde{B})$, the vector bundle induced over $\tilde{B}$ by $V(\tilde{P})$, the vertical subbundle of $T(\tilde{P}) ; V(\tilde{B})$ is isomorphic to the associated vector bundle $P \times_{H} \mathfrak{g} / \mathfrak{h} . B$ and $\tilde{P}$ are said to be soldered if $V(\tilde{B})$ and $T(\tilde{B})$ are isomorphic. If such a soldering exists then $T(B)$ is isomorphic to $V(\tilde{B})$. For any $X_{b} \in T_{b}(B)$ let $v_{b}\left(X_{b}\right) \in V(\tilde{P})$ be the vertical component of $\sigma\left(X_{b}\right)$ belonging to $T_{\sigma(b)}(\tilde{P})$; then, if there exists a Cartan connection $\Gamma$ for $P, v_{b}$ defines a bijective mapping between $T_{b}(B)$ and $V_{\sigma(b)}(\tilde{B})$ and $v$ is a soldering for $B$ and $\tilde{P}$ canonically associated to the Cartan connection $\Gamma$.

Definition 2. The 2-form $\Omega$ associated to a Cartan connection $\Gamma$ is given by the restriction to $P$ of the structure equation for $\Gamma$ considered as a usual connection on $P^{G}$, i.e.

$$
\Omega=d \omega+\frac{1}{2}[\omega, \omega]
$$

$\Omega$ is a $g$-valued 2 -form on $P$.

Since $P$ is parallelizable, the algebra of differentiable 1 -forms on $P$ is generated by $\omega$ and functions on $P$. Therefore if the components of $\omega$ with respect to a basis of $g$ are denoted by $w^{p}, \Omega$ can be written as

$$
\Omega=\frac{1}{2} \sum_{p, q} K_{p q} w^{p} \wedge w^{q}
$$

where each $K_{p q}$ is a $g$-valued function on $P$.

Let us also mention the following result concerning the automorphisms of a Cartan connection for $P$ which is also due to the property that $P$ is parallelizable [8]: the automorphisms of $P$ which preserve the 1-form $\omega$ generate a Lie group $\operatorname{Aut}(P, \omega)$, the dimension of which is at most equal to the dimension of $P$ $(\operatorname{dim} P=\operatorname{dim} G)$.

2.2 Symplectic Cartan connection. Now we want to explicitly study the properties of Cartan connections in the situation where the basis $B$ is a $(2 n+1)$-dimensional manifold, $G$ is the Lie group $\operatorname{Sp}(2(n+1), \mathbf{R})$ and $H$ is the semidirect product $W_{n}^{*}\left(3(\operatorname{Sp}(2 n, \mathbf{R}) \otimes \dot{\mathbf{R}})\right.$, so $G$ and $H$ will now be denoted by $L_{e}$ and $L^{0}$ as in $\S 1.1$.

Corresponding to the basis of the Lie algebra $\operatorname{sp}(2(n+1), \mathbf{R})$ given in $(7)$, let us introduce the following decomposition of the Cartan connection form $\omega$ :

$$
\omega=\sum_{\mu=0}^{2 n} w_{0^{\prime}}^{\mu} E_{\mu}^{0^{\prime}}+\frac{1}{2} \sum_{j, k=1}^{2 n} w_{j}^{k} E_{k}^{j}+w_{0}^{\sigma} E_{\sigma^{\alpha}}^{\sigma}+\sum_{\mu=0}^{2 n} w_{\mu}^{\sigma} E_{0^{\mu}}^{\mu}
$$

with the following conditions:

$$
w_{i+n}^{l+n}=-w_{l}^{i}, \quad w_{i+n}^{l}=w_{l+n}^{i}, \quad w_{i}^{l+n}=w_{l}^{i+n}, \quad i, l \in[1, n] .
$$


Properties P1-P3 of a Cartan connection explicitly become

Proposition 1. Let $\omega$ be a symplectic Cartan connection form whose components with respect to the basis (7) of the symplectic Lie algebra are defined in (15a, b); then

$\mathrm{P}^{\prime} 1$. For any vector field

$$
X=\frac{1}{2} \sum_{j, k=1}^{2 n} X_{j}^{k} E_{k}^{j}+X_{0}^{\sigma} E_{0}^{\sigma}+\sum_{\mu=0}^{2 n} X_{\mu} E_{0}^{\mu}
$$

on $L^{0}$ we have

$$
\begin{gathered}
w_{j}^{k}\left(X^{*}\right)=X_{j}^{k}, \quad w_{0}^{\sigma}\left(X^{*}\right)=X_{0}^{\sigma}, \quad j, k \in[1,2 n], \\
w_{\mu}^{\sigma}\left(X^{*}\right)=X_{\mu}, \quad \mu \in[0,2 n],
\end{gathered}
$$

which shows that, restricted to each fibre of $P,\left\{w_{j}^{k}, w_{0}^{0}, w_{\mu}^{0}\right\}$ is the Maurer-Cartan form on $L^{0}$. Moreover,

$$
w_{0^{\prime}}^{\mu}\left(X^{*}\right)=0 \text { for any } \mu \in[0,2 n]
$$

$\mathrm{P}^{\prime} 2$. The subspaces

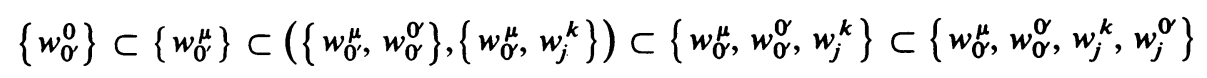

are stable under the action of $L^{0}$; this can be seen from

$$
\begin{aligned}
& R_{h}^{*}\left(w_{0^{\prime}}^{0}\right)=r^{-2} w_{0^{\prime}}^{0}, \\
& R_{h}^{*}\left(\bar{w}_{0^{\prime}}\right)=r^{-1} l^{-1} \bar{w}_{0^{\prime}}-r^{-2} J{ }^{t} \underline{u} w_{0^{\prime}}^{0}, \\
& R_{h}^{*}\left(w_{0^{\prime}}^{0^{\prime}}\right)=w_{0^{\prime}}^{\alpha}-r^{-1} \underline{u} l^{-1} \bar{w}_{0^{\prime}}-r^{-2} u_{0} w_{0^{\prime}}^{0}, \\
& R_{h}^{*}(w)=l^{-1} w l+r^{-1}\left(l^{-1} \bar{w}_{0} \otimes \underline{u}+J^{t} \underline{u} \otimes{ }^{t} \bar{w}_{0} J l\right) \\
& -r^{-2}{ }^{\prime} \underline{u} \otimes \underline{u} w_{0}^{0}, \\
& R_{h}^{*}\left(\underline{w}^{0^{\prime}}\right)=r \underline{w}^{0} l-\underline{u}^{-1} w l+\underline{u} w_{0}^{0} \\
& +r^{-1}\left(u_{0} \bar{w}_{0} J l-\underline{u}\left(\underline{u} l^{-1} \bar{w}_{0}\right)\right)-r^{-2} u_{0} \underline{u} w_{0}^{0}, \\
& R_{h}^{*}\left(w_{0}^{\sigma}\right)=r^{2} w_{0}^{0}-2 r \underline{u} l^{-1} J^{t} \underline{w}^{\sigma}-\underline{u} l^{-1} w l J^{t} \underline{u}+2 u_{0} w_{\sigma}^{\sigma} \\
& -2 r^{-1} u_{0}\left(\underline{u} l^{-1} \bar{w}_{0}\right)-r^{-2} u_{0}^{2} w_{0}^{0} .
\end{aligned}
$$

$P^{\prime} 3$. $V_{u}(P)$, the vector subspace of $T_{u}(P)$ defined by the $2 n+1$ equations $w_{0}^{\mu}\left(X_{u}\right)$ $=0$, is the subspace of vertical tangent vectors to $P$ at $u \in P$.

Besides, as a consequence of the above properties, any other set $\left\{\hat{w}_{\mu}^{\alpha}\right\}$ which, together with the set $s=\left\{w_{0}^{\mu}, w_{0}^{\alpha}, w_{j}^{k}\right\}$, gives rise to another Cartan connection, is given by

$$
w_{\mu}^{0^{\prime}}-\hat{w}_{\mu}^{0^{\prime}}=\sum_{\nu=0}^{2 n} A_{\mu \nu} w_{0}^{\nu}
$$

where the coefficients $\left\{A_{\mu \nu}\right\}$ are a set of $(2 n+1)^{2} C^{\infty}$ functions on $P$.

2.3 Structure equations, torsion and curvature of a symplectic Cartan connection. According to (13) the components of the 2-form $\Omega$ of a symplectic Cartan 
connection $\Gamma$ can be explicitly written as

$$
\begin{aligned}
& \Omega_{0^{\prime}}^{0}=d w_{0^{\prime}}^{0}+2 w_{0^{\prime}}^{0} \wedge w_{0^{\prime}}^{\sigma}+\sum w_{\sigma^{\prime}}^{l+n} \wedge w_{0^{\prime}}^{l}, \\
& \Omega_{0^{\prime}}^{j}=d w_{\sigma^{\prime}}^{j}-w_{\sigma^{\prime}}^{0} \wedge w_{n+j}^{\sigma}+\sum \tilde{w}_{l}^{j} \wedge w_{\alpha^{\prime}}^{l}, \\
& \Omega_{j}^{k}=d w_{j}^{k}-w_{j}^{0} \wedge w_{0}^{k}-w_{0}^{j+n} \wedge w_{k+n}^{0} \\
& +\sum \tilde{w}_{l}^{k} \wedge \tilde{w}_{j}^{l}+\delta_{j}^{k}\left(w_{0}^{0} \wedge w_{0}^{\alpha}-\sum w_{l}^{\alpha} \wedge w_{0}^{l}\right), \\
& \Omega_{0^{\prime}}^{0^{\prime}}=d w_{0^{\prime}}^{0}-w_{0}^{0} \wedge w_{0}^{\alpha}+\sum w_{l}^{0} \wedge w_{0}^{l}, \\
& \Omega_{j}^{\alpha}=d w_{j}^{\sigma^{\prime}}-w_{0}^{n+j} \wedge w_{0}^{\alpha}+\sum w_{l}^{\alpha} \wedge \tilde{w}_{j}^{l}, \\
& \Omega_{0}^{\alpha}=d w_{0}^{0^{\prime}}+2 w_{0}^{\alpha} \wedge w_{0}^{\alpha}+\sum w_{l}^{\alpha} \wedge w_{l+n}^{\alpha},
\end{aligned}
$$

where all the sums have to be performed over $l \in[1,2 n]$ and with the same conventions as the ones taken for the Maurer-Cartan form in $\S 1.2$.

According to the terminology introduced in [4] for the projective and conformal cases, $\left\{\Omega_{0}^{\mu}\right\}$ is called the torsion form and $\left\{\Omega_{j}^{k}, \Omega_{0}^{\alpha}, \Omega_{\mu}^{\alpha}\right\}$ the curvature form of the Cartan connection $\Gamma$.

Owing to the property $\mathrm{P}^{\prime} .1$ we deduce from the relations (18) that $\Omega$ is horizontal, more specifically relations (14) can be written uniquely in terms of $\left\{w_{0}^{\mu}\right\}$ and we can set

$$
\Omega^{(r)}=\frac{1}{2} \sum_{j, k=1}^{2 n} K_{j k}^{(r)} w_{0^{\prime}}^{j} \wedge w_{0^{\prime}}^{k}+\sum_{j=1}^{2 n} K_{j 0}^{(r)} w_{0^{j}}^{j} \wedge w_{0^{\prime}}^{0}, \quad\left(K_{j k}^{(r)}=-K_{k j}^{(r)}\right)
$$

where the index $\ldots{ }^{(r)}$ stands for the couple $\ldots{ }_{\beta}^{\alpha}$ with the following correspondences

$$
\begin{aligned}
\left(\alpha=0, \beta=0^{\prime}\right) \leftrightarrow r & =-2, \\
\left(\alpha \in[1,2 n], \beta=0^{\prime}\right) \leftrightarrow r & =-1, \\
(\alpha \in[0,2 n], \beta \in[0,2 n]) \text { and } \quad\left(\alpha=0^{\prime}, \beta=0^{\prime}\right) \leftrightarrow r & =0, \\
\left(\alpha=0^{\prime}, \beta \in[1,2 n]\right) \leftrightarrow r & =1, \\
\left(\alpha=0^{\prime}, \beta=0\right) \leftrightarrow r & =2 .
\end{aligned}
$$

There is no conservation property concerning all the components of the 2-form $\Omega$, i.e. in general $D \Omega \neq 0$ where $D$ denotes the exterior covariant differentiation. By applying the exterior differentiation to relations (18) and by collecting the terms which do not vanish when horizontal vector fields are applied one gets the following second set of structure equations

$$
\begin{aligned}
& D \Omega_{0^{\prime}}^{0}=-2 w_{0^{\prime}}^{0} \wedge \Omega_{0^{\prime}}^{0}-\sum\left(w_{0}^{l+n} \wedge \Omega_{\sigma^{\prime}}^{l}-\Omega_{\sigma^{\prime}}^{l+n} \wedge w_{\sigma^{\prime}}^{l}\right), \\
& D \Omega_{0^{\prime}}^{j}=w_{0^{\prime}}^{0} \wedge \Omega_{j+n}^{0}+\sum \Omega_{l}^{j} \wedge w_{0^{\prime}}^{l}, \\
& D \Omega_{j}^{k}=-w_{0^{\prime}}^{k} \wedge \Omega_{j}^{0}+w_{0}^{j+n} \wedge \Omega_{k+n}^{\alpha}-\delta_{j}^{k}\left(w_{0}^{0} \wedge \Omega_{0}^{0}+\sum \Omega_{l}^{0} \wedge w_{0}^{l}\right), \\
& D \Omega_{0^{\prime}}^{\sigma^{\prime}}=w_{0^{\prime}}^{0} \wedge \Omega_{0}^{\alpha}+\sum \Omega_{l}^{\alpha} \wedge w_{0^{\prime}}^{l} \text {, } \\
& D \Omega_{j}^{\alpha}=w_{0}^{n+j} \wedge \Omega_{0}^{\alpha} \text {, }
\end{aligned}
$$




$$
D \Omega_{0}^{0^{\prime}}=0 \text {, }
$$

with the convention $\Omega_{i+n}^{\sigma}=-\Omega_{i-n}^{0}$ and $\Omega_{\sigma}^{i+n}=-\Omega_{\sigma^{\prime}}^{i-n}$ for $i \in[n+1,2 n]$. Hence the component $\Omega_{0}^{0}$ only satisfies what is usually called a Bianchi identity.

2.4. In the projective and conformal cases it is known that the 2 -form $\Omega$ of the Cartan connection vanishes identically if the group $\operatorname{Aut}(P, \omega)$ has the maximal dimension, i.e. $\operatorname{dim} \operatorname{Aut}(P, \omega)=\operatorname{dim} P$ [9]. In the symplectic case this property is no longer valid because of the existence of the dilatation generated by $E_{\sigma}^{\sigma}$.

Let us set $s=\exp \left(\lambda E_{\sigma}^{\sigma}\right)$ belonging to $L^{0}$ and fix a point $u$ of $P$. Then if $\operatorname{dim} P=\operatorname{dim} \operatorname{Aut}(P, \omega)$, and due to the fact that $P$ is parallelizable [8], [9], there exists $\varphi_{\lambda} \in \operatorname{Aut}(P, \omega)$ such that $\varphi_{\lambda}(u)=R_{s}(u)$. Since $\operatorname{ad}\left(E_{\sigma}^{\sigma}\right)$ coincides with the multiplication by $r$ on $\mathrm{g}^{(r)}$, by (8), ad(s) is the multiplication by $e^{\lambda r}$ on $\mathrm{g}^{(r)}$. Hence

$$
R_{s}^{*}\left(\Omega^{(r)}\right)=\operatorname{ad}\left(s^{-1}\right) \Omega^{(r)}=e^{-\lambda r} \Omega^{(r)}
$$

and

$$
R_{s}^{*}\left(w^{(r)}\right)=\operatorname{ad}\left(s^{-1}\right) w^{(r)}=e^{-\lambda w^{(r)}} .
$$

But from (19) and (22) one gets

$$
R_{s}^{*}\left(\Omega^{(r)}\right)=\frac{1}{2} e^{2 \lambda} \sum_{j, k=1}^{2 n} R_{s}^{*}\left(K_{j k}^{(r)}\right) w_{0}^{j} \wedge w_{0}^{k}+e^{3 \lambda} \sum_{j=1}^{2 n} R_{s}^{*}\left(K_{j 0}^{(r)}\right) w_{0}^{j} \wedge w_{0}^{0},
$$

and by comparing with (21) one obtains

$$
\begin{aligned}
& R_{s}^{*}\left(K_{j k}^{(r)}\right)=e^{-(r+2) \lambda} K_{j k}^{(r)}, \\
& R_{s}^{*}\left(K_{j 0}^{(r)}\right)=e^{-(r+3) \lambda} K_{j 0}^{(r)} .
\end{aligned}
$$

On the other hand $\varphi_{\lambda}$ leaves $w^{(r)}$ and $\Omega^{(r)}$ invariant, so it leaves $K^{(r)}$ invariant. Then by using (24a) one can show that

$$
\begin{aligned}
K_{j k}^{(r)}(u) & =\varphi_{\lambda}^{*}\left(K_{j k}^{(r)}\right)(u)=K_{j k}^{(r)}\left(\varphi_{\lambda}(u)\right)=K_{j k}^{(r)}\left(R_{s}(u)\right) \\
& =R_{s}^{*}\left(K_{j k}^{(r)}\right)(u)=e^{-(r+2) \lambda} K_{j k}^{(r)}(u) .
\end{aligned}
$$

In the same way, by using $(24 b)$, one gets

$$
K_{j 0}^{(r)}(u)=e^{-(r+3) \lambda} K_{j 0}^{(r)}(u) .
$$

Hence (i) (25a) implies that $K_{j k}^{(r)}(u)=0$ for any $r \in[-1,2]$ and $j, k \in[1,2 n]$;

(ii) (25b) implies that $K_{j 0}^{(r)}(u)=0$ for any $r \in[-2,2]$ and $j \in[1,2 n]$ and, since $u$ is an arbitrary point of $P$, it has been proved that

Proposition 2. Let $\omega$ be the 1-form of a symplectic Cartan connection and $\Omega$ the corresponding 2-form. If $\operatorname{dim} \operatorname{Aut}(P, \omega)=\operatorname{dim} P$, the components $\Omega^{(-1),(0),(1),(2)}$ of $\Omega$ vanish identically, the component $\Omega^{(-2)}$ only can differ from zero and can be written

$$
\Omega_{0^{\prime}}^{0}=\frac{1}{2} \sum_{j, k=1}^{2 n} K_{0^{\prime} j k}^{0} w_{\sigma^{j}}^{j} \wedge w_{\sigma^{\prime}}^{k}
$$

2.5 Existence of a uniquely defined symplectic Cartan connection. Being given a set $s=\left\{w_{0}^{\mu}, w_{0}^{\alpha}, w_{j}^{k}\right\}$ of $2 n^{2}+3 n+2$ differential 1 -forms whose values in each point are linearly independent and which satisfy the properties $\mathbf{P}^{\prime} 1-\mathrm{P}^{\prime} 3$ of Proposition 1 , 
there exists at least one set $\left\{w_{\mu}^{\alpha}\right\}$ of $2 n+1$ independent 1 -forms such that (15a), (15b) define a Cartan connection form for $P$. This set $\left\{w_{\mu}^{\sigma}\right\}$ can be locally constructed as follows [13]: fixing a cross-section $\sigma: B \rightarrow P$ let us set $w_{\mu}^{0}(T)=0$ for every vector $T$ tangent to $\sigma(B)$. If $Z$ is an arbitrary tangent vector to $P$, it can be uniquely written as $Z=R_{h}(T)+Y$ where $h \in L^{0}, Y \in V(P)$ being the representative element of a unique fundamental vector field $Y^{*}$ on $P$ with $Y \in g^{(-2)}+$ $\mathfrak{g}^{(-1)}+\mathfrak{g}^{(0)}$. By using $\mathrm{P}^{\prime} 1, \mathrm{P}^{\prime} 2$, which in particular show that the set $s$ is invariant under the right action of $L^{0}$, the wanted set $\left\{w_{\mu}^{0}\right\}$ is then obtained by setting

$$
\omega(Z)=R_{h}^{*}(\omega(T))+Y .
$$

REMARK. A set $s$ being given, the $\Omega^{(-2)}$ component of the torsion form is fully determined; this is evident from (18a).

Now we want to show that $s$ being given, there exists a set $\left\{w_{\mu}^{\alpha}\right\}$ which completes $s$ to give rise to a Cartan connection $\Gamma$ uniquely defined owing to the following suitable constraints imposed on the curvature form

$$
\text { (a) } \sum_{k=1}^{2 n} K_{k j \mu}^{k}=0, \quad \text { (b) } \sum_{k=1}^{2 n} K_{k k \mu}^{j}=0, \quad \text { (c) } \sum_{k=1}^{2 n} K_{j k l}^{k}=0, \quad \mu \in[0,2 n] \text {. }
$$

We have seen that the sets of 1-forms $\left\{w_{\mu}^{\alpha}\right\}$ and $\left\{\hat{w}_{\mu}^{\alpha}\right\}$ which distinguish two Cartan connection forms $\omega$ and $\hat{\omega}$ built from the same set $s$ are related by (17). Let $\Omega$ and $\hat{\Omega}$ be the corresponding 2 -forms. $\Omega_{j}^{k}$ and $\hat{\Omega}_{j}^{k}$ can be written on one hand from their definition (18c) by taking (17) into account and with the following conventions: $A_{j+n, \mu}=-A_{j-n, \mu}, A_{\mu, j+n}=-A_{\mu, j-n}$ and $A_{j+n, k+n}=A_{j-n, k-n}$ for $j, k$ $\in[n+1,2 n]$; and, on the other hand, by means of the development (19) with coefficients $K^{(0)}$ and $\hat{K}^{(0)}$. Then by identifying the two so-obtained expressions one gets

$$
\begin{aligned}
K_{j k l}^{i}-\hat{K}_{j k l}^{i}= & -\delta_{j}^{i}\left(A_{k l}-A_{l k}\right)+\delta_{l}^{i} A_{j k}+\delta_{k}^{i} A_{j l} \\
& - \begin{cases}\delta_{l}^{j+n} A_{i+n, k}-\delta_{k}^{j+n} A_{i+n, l} & \text { if } j \in[1, n], \\
\delta_{k}^{j-n} A_{i+n, l}-\delta_{l}^{j-n} A_{i+n, k} & \text { if } j \in[n+1,2 n] ;\end{cases} \\
K_{j k 0}^{i}-\hat{K}_{j k 0}^{i}= & \delta_{j}^{i}\left(A_{k 0}-A_{0 k}\right)+\delta_{k}^{i} A_{j 0} \\
& - \begin{cases}\delta_{k}^{j+n} A_{i+n, 0} & \text { if } j \in[1, n], \\
-\delta_{k}^{j-n} A_{i+n, 0} & \text { if } j \in[n+1,2 n] .\end{cases}
\end{aligned}
$$

If the coefficients $K$ and $\hat{K}$ both satisfy (27) one can show that all the $A_{j \mu}$ and $A_{0 j}$ are zero, but $A_{00}$ cannot be determined by this way. Hence $w_{j}^{\sigma}=\hat{w}_{j}^{\sigma}$, but as we cannot conclude in what concerns $w_{0}^{\sigma}$ and $\hat{w}_{0}^{\sigma}$, at first sight it seems that (27) is not sufficient to lead to a unique Cartan connection. However this is not true and unicity and existence can be simultaneously proved as follows. Let $\hat{\Gamma}$ be any given Cartan connection, the 1 -form $\hat{\omega}$ of which contains the set $s$, i.e. $\hat{\omega}=\left\{s, \hat{w}_{\mu}^{\sigma}\right\}$. Let us consider another Cartan connection $\Gamma$ constructed from $\hat{\Gamma}$ as follows: its 1-form $\omega$ is given by $\omega=\left\{s, w_{\mu}^{0}\right\}$, where $w_{\mu}^{0}$ and $\hat{w}_{\mu}^{0}$ are related by (17) in such a way that the coefficients $K$ satisfy (27), i.e. the coefficients $A_{j \mu}$ and $A_{0 j}$ are linked to the $\hat{K}$ 's 
by means of (28a), (28b) in which conditions (27) are imposed on the $K$ 's. Explicitly one gets

$$
\begin{gathered}
A_{j k}=(2 n)^{-1} \sum \hat{K}_{j l k}^{l}-\left(4 n^{2}\right)^{-1} \sum \hat{K}_{l j k}^{l} \quad \text { if } j \neq k \pm n, \\
A_{j \pm n, j}=\frac{1}{2} \sum \hat{K}_{l j}^{j \pm n}, \\
A_{j 0}=\sum \hat{K}_{l l 0}^{j}-(2 n)^{-1} \sum \hat{K}_{l j 0}^{l}, \\
\left.A_{0 j}=\sum \hat{K}_{l l 0}^{j}-n^{-1} \sum \hat{K}_{l j 0}^{l} \quad \text { (all the sums being over } l \in[1,2 n]\right) .
\end{gathered}
$$

$A_{00}$ remaining undetermined, we are led to study the relationship between two Cartan connections $\Gamma_{1}$ and $\Gamma_{2}$ differing only by the coefficients $A_{00,1}$ and $A_{00,2}$ of $\hat{w}_{0}^{0}$ into the component $w^{(2)}$, i.e. such that $w_{0,1}^{\sigma}-w_{0,2}^{\sigma}=\left(A_{00,1}-A_{00,2}\right) \hat{w}_{0}^{0}$. (18a)(18d) show that the components $\Omega^{(-2)}, \Omega^{(-1)}$ and $\Omega^{(0)}$ of the 2-forms corresponding to $\Gamma_{1}$ and $\Gamma_{2}$ are identical; then, from (20d) one gets

$$
\left(A_{00,1}-A_{00,2}\right) w_{0}^{0} \wedge\left(d w_{0}^{0}-\sum w_{0}^{l} \wedge w_{0}^{n+l}\right)=0,
$$

which, by taking (18a) into account, can be written

$$
\left(A_{00,1}-A_{00,2}\right) w_{0}^{0} \wedge \Omega_{\sigma^{\prime}}^{0}=0 .
$$

If the Cartan connection $\Gamma$ is such that $\Omega_{0^{\prime}}^{0} \neq 0$, (31) leads to $A_{00,1}=A_{00,2}$, which implies $A_{00,1}=A_{00,2}=0$ since the $A_{00}$ 's are supposed arbitrary, hence $w_{0,1}^{0}=w_{0,2}^{0}$ $=\hat{w}_{0}^{0}$. If $\Omega_{0^{\prime}}^{0}=0,(31)$ is trivially satisfied. From $(18 \mathrm{e})$ one gets

$$
\Omega_{j, 1}^{\sigma}-\Omega_{j, 2}^{\sigma}=-\left(w_{0^{\prime}}^{n+j} \wedge w_{0^{\prime}}^{0}\right)\left(A_{00,1}-A_{00,2}\right) \text {. }
$$

Then (20e) and (18a) lead to

$$
\left(d w_{0^{\prime}}^{j}+2 w_{0^{\prime}}^{j} \wedge w_{0}^{\alpha}\right) \wedge w_{0^{\prime}}^{0}\left(A_{00,1}-A_{00,2}\right)=0,
$$

which, by taking (18b) into account, can be written

$$
\left(\Omega_{0^{\prime}}^{j}-\sum w_{l}^{j} \wedge w_{0^{\prime}}^{l}+2 w_{0}^{j} \wedge w_{\sigma^{\alpha}}^{\alpha}\right) \wedge w_{0}^{0}\left(A_{00,1}-A_{00,2}\right)=0 .
$$

In (34) the 2-form between brackets does not contain $w_{0^{\prime}}^{0}$ in factor so that one gets $A_{00,1}=A_{00,2}$ and again $w_{0,1}^{0}=w_{0,2}^{0}=\hat{w}_{0}^{0}$.

Hence it has been proved that

Proposition 3. From any Cartan connection given by its 1 -form $\hat{\omega}=\left\{s, \hat{w}_{\mu}^{\alpha}\right\}$ it is possible to construct a unique Cartan connection satisfying (29) whose 1-form $\omega=$ $\left\{s, w_{\mu}^{0}\right\}$ is such that $w_{\mu}^{\sigma}=\hat{w}_{\mu}^{\sigma}+\sum_{\nu=0}^{2 n} A_{\mu \nu} w_{0}^{\nu}$ where the coefficients $A_{\mu \nu}$ are given by (29a)-(29d) and $A_{00}=0$.

Note that $(27 a)$ is equivalent to

$$
\sum_{j=1}^{2 n} \Omega_{j}^{j}=0 ;
$$

then by taking the trace of $(20 \mathrm{c})$ one gets

$$
\sum_{\mu=0}^{2 n} \Omega_{\mu}^{0^{\prime}} \wedge w_{\sigma}^{\mu}=0 .
$$


In terms of the coefficients $K$ involved into the development (19), (36) gives rise to the following relations:

$$
\begin{aligned}
& K_{j k l}^{0}+K_{k l j}^{0}+K_{l j k}^{0}=0, \quad j \neq k, k \neq l, l \neq j, \\
& K_{0 j k}^{\sigma^{\prime}}+K_{j k 0}^{\sigma^{\prime}}-K_{k j 0}^{0^{\prime}}=0, \quad j \neq k \text {. }
\end{aligned}
$$

\section{Cartan structure for a contact manifold.}

3.1 Frames of second order contact and Cartan structures. Let $B$ be a $\nu$-dimensional differentiable manifold. Let $P^{r}(B)$ be the set of $r$-frames of $B$, i.e. the set of invertible $r$-jets $j_{0}^{r}(f) \in J_{0}^{r}\left(\mathbf{R}^{\nu}, B\right)$ of the diffeomorphisms $f: \mathbf{R}^{\nu} \rightarrow B$ with source $0 \in \mathbf{R}^{\nu} . P^{r}(B)$ is a principal fibre bundle over $B$ with structure group $G^{r}(\nu)$ and a natural projection $j_{0}^{r}(f) \rightarrow f(0) . G^{r}(\nu)$ is the group of invertible $r$-jets $j_{0}^{r}(f) \in$ $J_{0}^{r}\left(\mathbf{R}^{\nu}, \mathbf{R}^{\nu}\right)_{0}$ of the diffeomorphisms $f: \mathbf{R}^{\nu} \rightarrow \mathbf{R}^{\nu}$ with source 0 and target $f(0)=0$. By definition a reduced bundle $P$ of $P^{r}(B)$ with structure group $H$ subgroup of $G^{r}(\nu)$ is called an $H$-structure of degree $r$ on $B$.

From now on we shall be concerned with $P^{1}(B)$ and $P^{2}(B)$. The mapping $j_{0}^{2}(f) \rightarrow j_{0}^{1}(f)$ defines a bundle homomorphism of $P^{2}(B)$ into $P^{1}(B)$ and there is an exact sequence $0 \rightarrow N(\nu) \rightarrow G^{2}(\nu) \rightarrow G^{1}(\nu) \rightarrow 1$ where $N(\nu)$ is the abelian kernel of the natural homomorphism of $G^{2}(\nu)$ into $G^{1}(\nu)\left(G^{1}(\nu)\right.$ being isomorphic to $\mathrm{Gl}(\nu, \mathbf{R}))$. Let $\left\{x^{\rho}\right\}$ be a local chart of $B$ corresponding to the natural basis $\left\{e_{\rho}\right\}$ of $\mathbf{R}^{\nu}$. Being given $j_{0}^{2}(f) \in P^{2}(B)$ such that $f(0)$ is in the domain of the chart, a local coordinate system of $P^{2}(B)$ is given by $\left\{a^{\tau}, a_{\rho}^{\tau}, a_{\rho \sigma}^{\tau}\right\}$ where the $a$ 's are the coefficients of the unique polynomial representation of $j_{0}^{2}(f)$ of the form

$$
f(x)=\sum_{\rho, \sigma, \tau=0}^{\nu-1}\left(a^{\tau}+a_{\rho}^{\tau} x^{\rho}+a_{\rho \sigma}^{\tau} x^{\rho} x^{\sigma}\right) e_{\tau}
$$

where $a_{\rho \sigma}^{\tau}=a_{\sigma \rho}^{\tau}$ and $x=\sum_{\rho=0}^{\nu-1} x^{\rho} e_{\rho}$.

In the same manner, corresponding to the unique polynomial representation $\sum_{\rho, \sigma, \tau=0}^{\nu-1}\left(s_{\rho}^{\tau} x^{\rho}+s_{\rho \sigma}^{\tau} x^{\rho} x^{\sigma}\right) e_{\tau}$ of $j_{0}^{2}(f) \in G^{2}(\nu)$, a natural coordinate system in $G^{2}(\nu)$ is given by $\left\{s_{\rho}^{\tau}, s_{\rho \sigma}^{\tau}=s_{\sigma \rho}^{\tau}\right\}$, $\operatorname{dim} G^{2}(\nu)=\frac{1}{2} \nu^{2}(\nu+3)$. The group law in $G^{2}(\nu)$ is defined by the composition of jets as

$$
j_{0}^{2}(f) j_{0}^{2}\left(f^{\prime}\right)=j_{0}^{2}\left(f \circ f^{\prime}\right),
$$

and in terms of the natural coordinates it can be written as

$$
\left(s_{\rho}^{\tau}, s_{\rho \sigma}^{\tau}\right)\left(s_{\rho}^{\prime \tau}, s_{\rho \sigma}^{\prime \tau}\right)=\left(\sum_{\lambda=0}^{\nu-1} s_{\lambda}^{\tau} s_{\rho}^{\prime \lambda}, \quad \sum_{\lambda=0}^{\nu-1} s_{\lambda}^{\tau} s_{\rho \sigma}^{\prime \lambda}+\sum_{\lambda, \eta=0}^{\nu-1} s_{\lambda \eta}^{\tau} s_{\rho}^{\prime \lambda} s_{\sigma}^{\prime \eta}\right) .
$$

Locally the right action of $G^{2}(\nu)$ on $P^{2}(B)$ is given by

$$
\left(a^{\tau}, a_{\rho}^{\tau}, a_{\rho \sigma}^{\tau}\right)\left(s_{\rho}^{\tau}, s_{\rho \sigma}^{\tau}\right)=\left(a^{\tau}, \sum_{\lambda=0}^{\nu-1} a_{\lambda}^{\tau} s_{\rho}^{\lambda}, \sum_{\lambda=0}^{\nu-1} a_{\lambda}^{\tau} s_{\rho \sigma}^{\lambda}+\sum_{\lambda, \eta=0}^{\nu-1} a_{\lambda \eta}^{\tau} s_{\rho}^{\lambda} s_{\sigma}^{\eta}\right) .
$$

Similarly one can introduce the natural coordinates $\left\{a^{\tau}, a_{\rho}^{\tau}\right\}$ and $\left\{s_{\rho}^{\tau}\right\}$ in $P^{1}(B)$ and $G^{1}(\nu)$, respectively, so that the homomorphisms $P^{2}(B) \rightarrow P^{1}(B)$ and $G^{2}(\nu) \rightarrow$ $G^{1}(\nu)$ are given by $\left\{a^{\tau}, a_{\rho}^{\tau}, a_{\rho \sigma}^{\tau}\right\} \rightarrow\left\{a^{\tau}, a_{\rho}^{\tau}\right\}$ and $\left\{s_{\rho}^{\tau}, s_{\rho \sigma}^{\tau}\right\} \rightarrow\left\{s_{\rho}^{\tau}\right\}$, respectively.

$P^{r}\left(\mathbf{R}^{\nu}\right)(r>1)$ does not have a natural group structure but $P^{1}\left(\mathbf{R}^{\nu}\right)$ is isomorphic to the affine group $A(\nu, \mathbf{R})$, semidirect product of $\mathbf{R}^{\nu}$ and $\mathrm{Gl}(\nu, \mathbf{R})$. Let us denote 
by $e$ the 1-jet at 0 of the identity transformation of $\mathbf{R}^{\nu}$ which corresponds to the identity in $A(\nu, \mathbf{R})$ under this isomorphism. Then the tangent space $T_{e}\left(P^{1}\left(\mathbf{R}^{\nu}\right)\right)$ at $e$ is isomorphic to the Lie algebra of the affine group $a(\nu, \mathbf{R})=\mathbf{R}^{\nu} \square \mathrm{gl}(\nu, \mathbf{R})$.

Following [4] it is possible to introduce a canonical differential form $\theta$ on $P^{2}(B)$ which takes its values in $T_{e}\left(P^{1}\left(\mathbf{R}^{\nu}\right)\right)$, i.e. is an $a(\nu, \mathbf{R})$-valued 1 -form. $P^{1}(B)$ being locally homeomorphic to $P^{1}\left(\mathbf{R}^{\nu}\right),\left\{E_{\tau}^{\prime}=\left(\partial / \partial a^{\tau}\right)_{e}, E_{\tau}^{\prime \rho}=\left(\partial / \partial a_{\rho}^{\tau}\right)_{e}\right\}$ defines a basis for $a(\nu, \mathbf{R})$. Then locally $\theta$ can be written as

$$
\theta=\sum_{\tau=0}^{\nu-1} \theta^{\tau} E_{\tau}^{\prime}+\sum_{\tau, \rho=0}^{\nu-1} \theta_{\rho}^{\tau} E_{\tau}^{\prime \rho}
$$

with

$$
\begin{aligned}
& \theta^{\tau}=\sum_{\rho=0}^{\nu-1} b_{\rho}^{\tau} d a^{\rho}, \\
& \theta_{\rho}^{\tau}=\sum_{\sigma=0}^{\nu-1} b_{\sigma}^{\tau} d a_{\rho}^{\sigma}+\sum_{\lambda \sigma \eta=0}^{\nu-1} b_{\lambda}^{\tau} a_{\sigma \eta}^{\lambda} b_{\eta}^{\sigma} d a^{\eta}
\end{aligned}
$$

where $\left(b_{\sigma}^{\tau}\right)$ denotes the inverse of the matrix $\left(a_{\sigma}^{\tau}\right)$.

The components of the canonical form $\theta$ can be collected into a vector differential 1-form $\vartheta=\left(\theta^{0}, \bar{\theta}\right)$ where $\bar{\theta}=\left\{\theta^{\tau}\right\}, \tau \in[1, \nu-1]$, and a matrix of differential 1-forms

$$
\Theta=\left(\begin{array}{cc}
\theta_{0}^{0} & \underline{\theta}^{0} \\
\overline{\theta_{0}} & \tilde{\theta}
\end{array}\right)
$$

containing the row $1 \times(\nu-1)$ matrix $\underline{\theta}^{0}=\left\{\theta_{\tau}^{0}\right\}$, the column $(\nu-1) \times 1$ matrix $\bar{\theta}_{0}=\left\{\theta_{0}^{\tau}\right\}$ and the $(\nu-1) \times(\nu-1)$ matrix $\tilde{\theta}=\left(\theta_{\rho}^{\tau}\right)$. Under the action of $s \in$ $G^{2}(\nu)$ the canonical form transforms as

$$
R_{s}^{*}(\theta)=\operatorname{ad}\left(s^{-1}\right) \theta
$$

and locally from (41) and (42b), (42c) one gets

$$
\begin{aligned}
& R_{s}^{*}(\vartheta)=s^{-1} \boldsymbol{\vartheta} \\
& R_{s}^{*}(\Theta)=s^{-1} d s+s^{-1} \Theta s-\Xi,
\end{aligned}
$$

where $\Xi$ is the matrix $\left(\sum_{\lambda \sigma \eta=0}^{\nu-1} t_{\lambda}^{\tau} s_{\sigma \rho}^{\lambda} t_{\eta}^{\sigma} \theta^{\eta}\right), t_{\lambda}^{\tau}$ denoting the elements of the inverse of the $\nu \times \nu$ matrix $s=\left(s_{\lambda}^{\tau}\right)$.

The canonical form on $P^{2}(B)$ possesses the following important property:

$$
d \theta^{\tau}=-\sum_{\lambda=0}^{\nu-1} \theta_{\lambda}^{\tau} \wedge \theta^{\lambda} .
$$

By using the above recollected notions a modern and precise description of the spaces constructed by Cartan [2] has been given in [4]; such spaces can be called Cartan structures and can be defined as follows:

Definition 3. Let $B$ be a $\nu$-dimensional differentiable manifold and $P^{2}(B)$ the bundle of 2-frames over $B$ with structure group $G^{2}(\nu)$. A $G / H$ Cartan structure on $B$ is an $H$-structure of degree 2 on $B$ with a connection $\Gamma$ which is the unique 
Cartan connection with respect to $G \supset H(\operatorname{dim} G / H=\nu)$ constructed by supplementing as in Proposition 3 of $\$ 2.5$ a set $s$ of independent differential 1-forms expressed in terms of the restriction to $P$ of the canonical form $\theta$ on $P^{2}(B)$.

Two well-known examples of $G / H$ Cartan structures are the projective structure with $G=\operatorname{PGL}(\nu+1, \mathbf{R}), \quad H=\mathbf{R}^{\nu}(3(\operatorname{SL}(\nu, \mathbf{R}) \otimes \dot{\mathbf{R}}), \operatorname{dim} B=\nu$ and the conformal structure with $G=O(\nu+1,1), H=\mathbf{R}^{\nu}$ ( ) $C O(\nu), \operatorname{dim} B=\nu$. In both cases $H$ is a semidirect product, of which the invariant subgroup $\mathbf{R}^{\nu}$ is the kernel of the linear isotropy representation. Consequently $\mathbf{R}^{\nu}$ appears as a subgroup of $N(\nu)$.

3.2 Contact structure on a $(2 n+1)$-dimensional manifold.

Definition 4. Let $L_{e}$ and $L^{0}$ be as in \$1.1. An $L_{e} / L^{0}$ Cartan structure over a $(2 n+1)$-dimensional manifold $B$ will be called a contact structure on $B$.

As a first step we have to show that $L^{0} \approx W_{n}$ (3) $\operatorname{CSp}(2 n, \mathbf{R})$ can be realized as a subgroup of $G^{2}(2 n+1)$. Let us consider the restriction to an element $m \in L^{0}$ of the linear fractional transformation of $\mathbf{R}^{2 n+1},\left(\bar{z}, z_{0}\right) \mapsto f_{m}\left(\bar{z}, z_{0}\right)=\left(\bar{z}, \hat{z}_{0}\right)$, which is obtained by setting $\bar{v}=0, v_{0}=0$ into (11). Then the coefficients of the unique polynomial representation of $j_{0}^{2}\left(f_{m}\right) \in J_{0}^{2}\left(\mathbf{R}^{2 n+1}, \mathbf{R}^{2 n+1}\right)_{0}$ are given by

$$
\begin{gathered}
s_{0}^{0}=r^{2}, \quad s_{j}^{0}=0, \quad s_{j}^{k}=r l_{j}^{k}, \quad s_{0}^{k}=r\left(U^{t} \underline{u}\right)^{k}, \quad j, k \in[1,2 n], \\
s_{\rho \sigma}^{\tau}=-\frac{1}{2}\left(s_{\rho}^{\tau} u_{\sigma}+s_{\sigma}^{\tau} u_{\rho}\right)-\frac{1}{2} u_{0}\left(\delta_{\rho}^{0} s_{\sigma}^{\tau}+\delta_{\sigma}^{0} s_{\rho}^{\tau}\right), \quad \tau, \rho, \sigma \in[0,2 n] .
\end{gathered}
$$

It is easy to verify that, with the above expressions of $\left\{s_{\rho}^{\tau}, s_{\rho \sigma}^{\tau}\right\}$, the group law of $L^{0}$ given in (3) is recovered by using (40). Hence one has

Proposition 4. For each element $m \in L^{0}$ given by $\left(\overline{0}, 0, l, r, \underline{u}, u_{0}\right)$ in terms of the coordinate system chosen for $L_{e}$, let $f_{m}$ be the linear fractional transformation of $\mathbf{R}^{2 n+1}$ given by

$$
\overline{\hat{z}}=r\left(1+\underline{u} \bar{z}+u_{0} z_{0}\right)^{-1}\left(J^{t} \underline{u}^{t} z_{0}+l \bar{z}\right), \quad \hat{z}_{0}=r^{2}\left(1+\underline{u} \bar{z}+u_{0} z_{0}\right)^{-1} z_{0} .
$$

Then the mapping $m \rightarrow j_{0}^{2}\left(f_{m}\right)$ defines a faithful representation of $L^{0}$ into $G^{2}(2 n+1)$ which can be described by the following diagram:

$$
\begin{aligned}
& 0 \rightarrow N(2 n+1) \rightarrow G^{2}(2 n+1) \rightarrow G^{1}(2 n+1) \rightarrow 1
\end{aligned}
$$

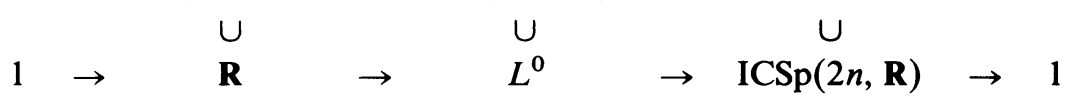

(This is evident from the explicit expressions of the coordinates of the image of $m$ into $G^{2}(2 n+1)$ given by (46).)

The lower exact sequence which makes $L^{0}$ appear as a one-parameter central extension of the inhomogeneous conformal symplectic group $\operatorname{ICSp}(2 n, \mathbf{R})$ has already been described in $\$ 1.3$. Let $L^{1}$ denote the linear isotropy group $\operatorname{ICSp}(2 n, \mathbf{R})$; then $L^{1}$ embedded into $G^{1}(2 n+1)$ can be considered as the subgroup of $G^{2}(2 n+1)$ consisting of elements $\left(s_{\rho}^{\tau}, 0\right), \tau, \rho \in[0,2 n]$, and the section $k$ which defines the central extension (cf. \$1.3) is explicitly given by $\left(s_{\rho}^{\tau}, 0\right) \mapsto\left(s_{\rho}^{\tau}, s_{\rho o}^{\tau}\right)$.

If $P$ denotes an $L^{0}$-structure of degree 2 on a $(2 n+1)$-dimensional manifold $B$, then $P / \operatorname{ker}(p)$ is a subbundle $Q$ of $P^{1}(B)$ with $L^{1} \subset G^{1}(2 n+1)$ as structure group. (Note that $\operatorname{ker}(p) \approx \mathbf{R}$ from the very definition of the exact sequence 
written in $\S 1$.3.) Conversely let $Q$ be an $L^{1}$-structure of degree 1 and $k$ an injective homomorphism from $L^{1} \approx \operatorname{ICSp}(2 n, \mathbf{R})$ into $L^{0} \approx W_{n}$ (5) $\operatorname{CSp}(2 n, \mathbf{R}) . L^{1}$ acts on the left on $L^{0}$ as follows: $(a, m) \mapsto k(a) m, a \in L^{1}, m \in L^{0}$. So we can introduce the associated fibre bundle $\left(Q \times L^{9}\right) / L^{1}=Q_{k}$, which is a principal fibre bundle, the $k$-extension of $Q$, with respect to the right action of $L^{0}$ over $Q_{k}$ given by

$$
\left(\left(q^{\cdot} m\right), m^{\prime}\right) \mapsto q^{\cdot}\left(m m^{\prime}\right), \quad q \in Q, m, m^{\prime} \in L^{0},
$$

$q^{\cdot} m$ denoting the class of $(q, m)$ into $Q_{k}$. If $k$ is chosen as above then the $k$-extension of $Q$ will be an $L^{0}$-structure of degree 2 . Accordingly there is a natural mapping between the bundles $P^{2}(B) / L^{1}$ and $P^{2}(B) / L^{0}$ associated to $Q$ and $P$ respectively.

In the second step, being given an $L^{0}$-structure $P$ of degree 2 on a $(2 n+1)$ dimensional manifold $B$, we have to construct a Cartan connection on $P$ in a canonical way. Let us denote by $\boldsymbol{\theta}^{\prime}=\left\{\boldsymbol{\vartheta}^{\prime}, \Theta^{\prime}\right\}$ the restriction to the $L^{\mathbf{0}}$-subbundle of $P^{2}(B)$ of the canonical 1-form $\theta$. Explicitly one gets

$$
\boldsymbol{\vartheta}^{\prime}=\left\{\boldsymbol{\theta}^{0}, \bar{\theta}\right\}, \quad \Theta^{\prime}=\left[\begin{array}{cc}
\theta_{0}^{0} & -{ }^{t} \bar{\theta} J \\
\overline{\theta_{0}} & \frac{1}{2}\left(\tilde{\theta}+J^{t} \tilde{\theta} J\right)
\end{array}\right] .
$$

By comparing over any open set of $B$ the right actions of the structure group $L^{0}$, on one hand on the 1-form Cartan connection by using (16a)-(16d), on the other hand on the canonical 1 -form $\theta^{\prime}$ by using (44a), (44b), one verifies that $w_{0}^{0}, \bar{w}_{0}, w_{0}^{\alpha}, w$ transform like $\theta^{0}, \bar{\theta},-\frac{1}{2} \theta_{0}^{0}, \frac{1}{2}\left(\tilde{\theta}+J^{i} \tilde{\theta} J\right)$, respectively. Consequently one takes $s=\left\{\theta^{0}, \bar{\theta},-\frac{1}{2} \theta_{0}^{0}, \frac{1}{2}\left(\tilde{\theta}+J^{i} \tilde{\theta} J\right)\right\}$ as the set of differential 1-forms which can be supplemented to give rise to the unique symplectic 1-form connection as described in $\$ 2.5$, so we can state the following definition.

Definition 5. The unique symplectic 1 -form connection obtained by supplementing the set $s$ of differential 1 -forms $s=\left\{\theta^{0}, \bar{\theta},-\frac{1}{2} \theta_{0}^{0}, \frac{1}{2}\left(\tilde{\theta}+J^{t} \tilde{\theta} J\right)\right\}$ as described in Proposition $3, \S 2.5$, is called the "normal" symplectic Cartan connection on the $L^{0}$ principal bundle $P$.

Contrary to what happens for the projective and conformal Cartan connections in general the "normal" symplectic Cartan connection has not a vanishing torsion since the right-hand sides of (18.a) and (18.b) do not vanish by taking relation (45) into account.

Proposition 5. Let $P$ be an $L^{0}$-principal bundle over $a(2 n+1)$-dimensional manifold $B$ considered as a reduced subbundle of $P^{2}(B)$. There is a "normal" symplectic Cartan connection on $P$ such that the coefficients $K$ introduced in the decomposition (19) satisfy the following conditions:

$$
\sum_{j=1}^{2 n} K_{j k \mu}^{j} \doteq 0, \quad \sum_{j=1}^{2 n} K_{j j \mu}^{k}=0, \quad \sum_{j=1}^{2 n} K_{k j l}^{j}=0 .
$$

The curvature of such a connection has the following properties:

(i) $\sum_{j=1}^{2 n} \Omega_{j}^{j}=0$ or, equivalently, $\sum_{j=1}^{2 n} K_{j k \mu}^{j}=0$;

(ii) $\sum_{\mu=0}^{2 n} \Omega_{\mu}^{0} \wedge w_{0}^{\mu}=0$ or, equivalently,

$$
K_{j k l}^{\alpha}+K_{k l j}^{\alpha}+K_{l j k}^{\alpha}=0 \text { and } K_{0 j k}^{0}+K_{j k 0}^{\alpha}-K_{k j 0}^{\alpha}=0 ;
$$


In this section it has been shown that being given a $(2 n+1)$-dimensional manifold $B$ it is possible to look for an $L^{0}$-structure of degree 2 on $B$ (Proposition 4 ); then the "normal" symplectic connection can be defined on $P$ (Proposition 5). Therefore according to Definitions 3-4, any $L^{0}$-structure of degree 2 over a $(2 n+1)$-dimensional manifold $B$ can be considered as an $L_{e} / L^{0}$ Cartan structure or a contact structure on $B$.

$L_{e} / L^{0}$ itself possesses a natural contact structure in the sense of Definition 4 . It is given by the subbundle $P_{0}$ of $P^{2}\left(L_{e} / L^{9}\right)$ consisting of invertible elements of the form $j_{0}^{2}(f) \in J_{0}^{2}\left(\mathbf{R}^{2 n+1}, L_{e} / L^{0}\right)$ where $f: \mathbf{R}^{2 n+1} \rightarrow L_{e} / L^{0}$ is the restriction of a contact transformation on $L_{e} / L^{0}$ obtained by fixing an embedding of $\mathbf{R}^{2 n+1}$ in $L_{e} / L^{0}$ in a neighbourhood of the origin 0 . It is easy to check from the action of $L_{e}$ given by (11) that $\alpha^{0}$ (defined in $\S 1.3$ ) is kept invariant under the transformations $f \in L^{0}$ parametrized by $\left(\bar{v}, v_{0}, l, 1, \underline{0}, 0\right)$, and $\alpha^{0} \rightarrow D^{2} \alpha^{0}$ under the transformations $f \in W_{n}^{*}$ ( ) $\dot{\mathbf{R}}$ parametrized by $\left(\overline{0}, 0,1_{2 n}, r, \underline{u}, u_{0}\right)$. Then one has a bundle isomorphism between $L_{e}$ as a principal $L^{0}$-bundle over $L_{e} / L^{0}$ and $P_{0}$ over $L_{e} / L^{0}$ compatible with the isomorphic embedding of $L^{0}$ into $G^{2}(2 n+1)$. The "normal" symplectic connection associated with $P_{0}$ corresponds to the Maurer-Cartan form of $\operatorname{Sp}(2 n+2, \mathbf{R})$, so it has no curvature (and no torsion).

3.3 Contact isomorphisms and flat contact structures.

Definition 6. Let $P$ and $P^{\prime}$ be two contact structures on two $(2 n+1)$ dimensional manifolds $B$ and $B^{\prime}$, respectively. A (local) diffeomorphism $\psi: B \rightarrow B^{\prime}$ induces a local isomorphism $\psi_{*}$ between $P^{2}(B)$ and $P^{2}\left(B^{\prime}\right)$. If $\psi_{*}$ sends $P$ into $P^{\prime}$ it is then necessarily a bundle isomorphism and $\psi$ is called a (local) contact isomorphism of $B$ into $B^{\prime}$. If $B=B^{\prime}$ and $P=P^{\prime}, \psi$ will be called a contact transformation.

From the property of $\operatorname{Aut}(P, \omega)$ mentioned in $\$ 2.1$ and the unicity of the "normal" Cartan connection one gets

Proposition 6. If $P$ is a contact structure on a $(2 n+1)$-dimensional manifold $B$ then the group of contact transformations is a Lie transformation group of dimension $\leqslant \operatorname{dim}(P)=(n+1)(2 n+3)$.

Note that the group of contact transformations (in the sense of Definition 6) of $L_{e} / L^{0}$ is precisely the symplectic group $\operatorname{Sp}(2 n+2, \mathbf{R})$.

But let us recall that, from Proposition 2, the existence of $L^{0}$-structures of second degree with a Cartan connection possessing an automorphism group of maximal dimension and a nonzero component $\Omega_{0}^{0}$ of the torsion is not excluded.

Finally the notion of contact isomorphism is used to give the following definition.

Definition 7. A contact structure $P$ over a $(2 n+1)$-dimensional manifold $B$ is called flat if for each point of $B$ there is a neighbourhood $U$ and a local contact isomorphism of $U$ onto an open subset of $L_{e} / L^{0}$.

Obviously the "normal" symplectic connection of a flat Cartan structure has a vanishing curvature.

3.4 Contact structure on a contact manifold. Now let us consider a $(2 n+1)$ dimensional contact manifold $(C, \alpha)$. Let $\mathbf{R}^{2 n+1}$ be the standard fibre of the 
tangent bundle $T(C)$ associated to $P^{1}(C)$. It is well known that any element $p$ belonging to $P^{1}(C)$ over $x \in C$ can be considered as a mapping of $\mathbf{R}^{2 n+1}$ onto $T_{x}(C): \xi \rightarrow p \xi=\xi_{x}$. The exterior derivative of $\alpha$ is a closed two-form of maximal rank $2 n$, hence at each point $x \in C$, $d \alpha$ defines a nondegenerate skew-symmetric bilinear form on the set $\left\{\xi_{x} \in T_{x}(C) \mid \alpha\left(\xi_{x}\right)=0\right\}$. Then it is possible to associate with $d \alpha$ a bilinear form (, ) on $\mathbf{R}^{2 n+1}$ defined by

$$
\left(\xi, \xi^{\prime}\right)=\left(p^{-1} \xi_{x}, p^{-1} \xi_{x}^{\prime}\right)=d \alpha\left(\xi_{x}, \xi_{x}^{\prime}\right) .
$$

If $\tilde{J}$ denotes the linear transformation of $\mathbf{R}^{2 n+1}$ given by the matrix $\left(\begin{array}{l}J \\ 0\end{array} 0\right)$, then the above defined bilinear form can be written as

$$
\left(\xi, \xi^{\prime}\right)={ }^{\prime} \xi \tilde{J} \xi^{\prime},
$$

where $\xi$ is written as a column $(2 n+1) \times 1$ matrix and ${ }^{t} \xi$ as a row $1 \times(2 n+1)$ matrix. Obviously ( , ) is invariant by the subgroup of $\operatorname{GL}(2 n+1, \mathbf{R})$ given by $\left\{\left.M \in \mathrm{GL}(2 n+1, \mathbf{R})\right|^{t} M \tilde{J} M=\tilde{J}\right\}$. It is easy to verify that this subgroup is nothing else than the inhomogeneous conformal symplectic group $\operatorname{ICSp}(2 n, \mathbf{R})$. The invariance of $($,$) by \operatorname{ICSp}(2 n, \mathbf{R})$ implies that $(50)$ is independent of the choice of $p$ modulo a right action of an element of $\operatorname{ICSp}(2 n, \mathbf{R})$ as a subgroup of $\mathrm{GL}(2 n+1, \mathbf{R})$ into the fibre bundle of linear frames over $C$, i.e. it leads to a reduction of $P^{1}(C)$ to an $L^{1}$-structure. So one gets the following proposition.

Proposition 7. (1) To a given contact manifold $(C, \alpha)$ can be associated in a canonical way an $L^{1}$-structure $Q$ on $C$ (of course, the converse is not in general true).

(2) The $k$-extension of $Q(c f . \S 3.2)$ associates an $L^{0}$-structure of degree 2 and hence a contact structure to any contact manifold $(C, \alpha)$.

Let us consider the bundle $P^{2}(C) / L^{1}$ with fibre $G^{2}(2 n+1) / L^{1}$ associated to the principal $L^{1}$ bundle $Q$. Let $\left(a^{\tau}, a_{\rho}^{\tau}, a_{\rho o}^{\tau}\right)$ be the local coordinate system in $P^{2}(C)$ induced from a local coordinate system in $C$ as in 3.1. We introduce a local coordinate system $\left(b^{\tau}, b_{\rho}^{\tau}, b_{\rho \sigma}^{\tau}\right)$ in $P^{2}(C) / L^{1}$ in such a way that the projection $P^{2}(C) \rightarrow P^{2}(C) / L^{1}$ is given by the equations

$$
b^{\tau}=a^{\tau}, \quad b_{\rho}^{\tau}, \quad b_{\rho \sigma}^{\tau}=\sum a_{\lambda \eta}^{\tau}\left(a^{-1}\right)_{\rho}^{\lambda}\left(a^{-1}\right)_{\sigma}^{\eta},
$$

where $\left(a^{-1}\right)_{\rho}^{\lambda}$ denotes the element $\ldots{ }_{\rho}^{\lambda}$ of the inverse of the matrix $a=\left(a_{\sigma}^{\eta}\right)$. Then a cross-section $\Gamma: C \rightarrow P^{2}(C) / L^{1}$ is given locally by $b^{\tau}=x^{\tau}$ and a set of functions $b_{\rho \sigma}^{\tau}=-\Gamma_{\rho \sigma}^{\tau}(x)$ with $\Gamma_{\rho \sigma}^{\tau}=\Gamma_{\sigma \rho}^{\tau}, b_{\rho}^{\tau}$ unspecified. Under the action of the group $G^{2}(2 n+1)$ on the fibre $G^{2}(2 n+1) / L^{1}$ corresponding to a change of coordinate system of $C$, one verifies that the functions $\Gamma_{\rho \sigma}^{\tau}$ behave as Christoffel's symbols must. This proves

Proposition 8. The cross-sections $\Gamma: C \rightarrow P^{2}(C) / L^{1}$ are in one-to-one correspondence with the $\operatorname{ICSp}(2 n, \mathbf{R})$-connections without torsion. By $\operatorname{ICSp}(2 n, \mathbf{R})$-connection we mean the restriction onto $Q$ of an affine connection on $C$ with value in the Lie algebra of $\operatorname{ICSp}(2 n, \mathbf{R})$.

On the other hand from the reduction theorem we know that the cross-sections $\tilde{\Gamma}: C \rightarrow P^{2}(C) / L^{0}$ are in one-to-one correspondence with the reductions of 
$G^{2}(2 n+1)$ to the structure group $L^{0}$, i.e. with the $L^{0}$-structures $P$ of degree 2 and, consequently, with the contact structures. Then from Proposition 8 one gets

Proposition 9. Every $\operatorname{ICSp}(2 n, \mathbf{R})$-connection without torsion corresponding to a cross-section $\Gamma: C \rightarrow P^{2}(C) / L^{1}$ composed with the natural mapping $P^{2}(C) / L^{1} \rightarrow$ $P^{2}(C) / L^{0}(\$ 3.2)$ gives rise to a contact structure which is said to be associated with the $\operatorname{ICSp}(2 n, \mathbf{R})$-connection.

Definition 8. Two $\operatorname{ICSp}(2 n, \mathbf{R})$-connections $\Gamma$ and $\Gamma^{\prime}$ on $Q$ without torsion are called $L^{0}$-equivalent if they give rise to the same Cartan structure. This is an equivalence relation and each equivalence class of $\operatorname{ICSp}(2 n, \mathbf{R})$-connections on $Q$ without torsion uniquely defines an associated contact structure.

Proposition 10. Two torsion free $L^{1}$-connections on an $L^{1}$-structure $Q$ (of degree 1) are $L^{0}$-equivalent if and only if there is an $\mathbf{R}^{2 n+1}$-valued function $\xi$ on $Q$ such that the connection forms $\bar{\omega}$ and $\bar{\omega}^{\prime}$ are related by

$$
\bar{\omega}^{\prime}-\bar{\omega}=\vartheta^{\prime} \otimes \xi+\xi \vartheta^{\prime} \mathbf{1}_{2 n+1} .
$$

Let $\Gamma$ and $\Gamma^{\prime}$ be two cross-sections, $C \rightarrow P^{2}(C) / L^{1}$ given in the above-considered coordinate system by $\left(b^{\tau}, b_{\rho}^{\tau}, b_{\rho \sigma}^{\tau}\right)$ and $\left(b^{\tau}, b_{\rho}^{\tau}, b_{\rho \sigma}^{\prime \tau}\right)$, i.e. two torsion free $L^{1}$-connections, and let $\bar{\omega}$ and $\bar{\omega}^{\prime}$ be the corresponding connection forms on $Q$. It is easy to check that $b_{\rho \sigma}^{\tau}$ given by (52) is invariant under the action induced by an element $\left(s_{\rho}^{\tau}, 0\right)$ of $L^{1} \subset G^{2}(2 n+1)$. But under the action of $\left(s_{\rho}^{\tau}, s_{\rho \sigma}^{\tau}\right)$ belonging to $L^{0}$ one gets $b_{\rho \sigma}^{\tau} \rightarrow \hat{b}_{\rho \sigma}^{\tau}=b_{\rho \sigma}^{\tau}-\frac{1}{2}\left(\delta_{\sigma}^{\tau} \varphi_{\rho}+\delta_{\rho}^{\tau} \varphi_{\sigma}\right)$ if we put $\varphi=u s^{-1} a^{-1}$, where $u$ is the row $\left(u_{0}, \underline{u}\right)$ and $s$ is the matrix $\left(s_{\rho}^{\tau}\right)$ given by (45). So $\Gamma$ and $\Gamma^{\prime}$ give rise to the same section $\tilde{\Gamma}: C \rightarrow P^{2}(C) / L^{0}$, when composed with the natural mapping $P^{2}(C) / L^{1} \rightarrow P^{2}(C) / L^{0}$, if and only if there is an $\mathbf{R}^{2 n+1}$-valued function $\eta$ on $C$ such that

$$
\Gamma_{\rho \sigma}^{\prime \tau}-\Gamma_{\rho \sigma}^{\tau}=\delta_{\rho}^{\tau} \eta_{\sigma}+\delta_{\sigma}^{\tau} \eta_{\rho}
$$

but in terms of the local coordinate system this relation is equivalent to

$$
w_{\rho}^{\prime \tau}-w_{\rho}^{\tau}=\vartheta^{\prime \tau} \xi_{\rho}+\delta_{\rho}^{\tau} \xi \vartheta^{\prime}
$$

where $\vartheta^{\prime}$ is the canonical $\mathbf{R}^{2 n+1}$-valued form on $Q$. Q.E.D.

\section{REFERENCES}

1. G. Burdet and M. Perrin, Realizations of the central extension of the inhomogeneous symplectic algebra as time dependent invariance algebras of nonrelativistic quantum systems, J. Mathematical Phys. 16 (1975), 1692-1703, Appendix A.

2. E. Cartan, Oeuvres complètes. Partie III, Vol. 1, Gauthier-Villars, Paris, 1955.

3. Ch. Ehresmann, Les connexions infinitésimales dans un espace fibré différentiable, Colloq. Topologie (Brussels, 1950), Thone, Liège; Masson, Paris, 1951, pp. 29-55.

4. S. Kobayashi, Transformation groups in differential geometry, Springer-Verlag, Berlin and New York, 1972.

5. , ibid., p. 28.

6. op. cit., p. 127.

7.

8. op. cit., p. 15.

9. op. cit., p. 130.

10. __ op. cit., p. 137.

Centre National de la Recherche Scientifique, Université de Duon, F21004 Dijon, France 